\title{
Surface Modifications of Cellulose Acetate Film for the Application of Face Shield
}

\author{
Sunwoo E. Lee \\ Thomas Jefferson High School for Science and Technology, Alexandria, Virginia, USA \\ Email: sunnyelee1@gmail.com
}

How to cite this paper: Lee, S.E. (2020) Surface Modifications of Cellulose Acetate Film for the Application of Face Shield. Journal of Materials Science and Chemical Engineering, 8, 41-45.

https://doi.org/10.4236/msce.2020.88004

Received: July 18, 2020

Accepted: August 24, 2020

Published: August 27, 2020

Copyright ( 2020 by author(s) and Scientific Research Publishing Inc. This work is licensed under the Creative Commons Attribution International License (CC BY 4.0).

http://creativecommons.org/licenses/by/4.0/

(c) (i) Open Access

\begin{abstract}
During the COVID-19 pandemic, personal protective equipment (PPE) has become crucial to protect humans from the transmission of the virus. The face shield is a simple and effective PPE to prevent the viral and bacterial contact. Since COVID-19 is known to be spread via respiratory droplets, the face shield has become increasingly important PPE. However, the common materials used in face shields are synthetic, environmentally unfriendly polymers, which cause an accumulation of plastic waste once disposed. Cellulose acetate (CA) can be used as an alternative for face shield films due to its ability to decompose safely in the environment; however, pristine CA cannot serve as an effective face shield due to its low hydrophobicity. In this research, the somewhat hydrophilic character of CA with a water contact angle of $55^{\circ}$ is experimented on: hexamethyldisilazane (HMDS) is utilized to improve the hydrophobicity of CA up to a water contact angle of $77^{\circ}$. After the oxidization of the surface of CA via oxygen plasma, implementing HMDS shows a significant increase in hydrophobicity of the film.
\end{abstract}

\section{Keywords}

Face Shield, Cellulose Acetate, Hydrophobicity

\section{Introduction}

The current COVID-19 pandemic has greatly impacted our daily lives. As of July 15, 2020 in the United States [1], the virus has infected more than 3.5 million people and caused nearly 140 thousand deaths. While States are gradually reopening local stores and restaurants since June 2020, wearing PPE such as face masks and gloves has been strongly recommended or is even mandatory in order to go to public places. PPE is particularly significant to protect healthcare work- 
ers and workers in public and commercial service sectors. For example, the nurses who are treating COVID-19 patients must wear both medical masks and face shields in order to treat them. As long as the pandemic continues, the demand for face shields is expected to increase.

The face shield is a simple, easily accessible item to cover up the face from unsanitary substances in the environment. The main component (shield) is made of polymer films; polyethylene terephthalate (PET), polycarbonates (PC), and polyvinylchlorides (PVC) are widely used as face shields. These polymers are major materials in the current market. Although these successfully protect saliva droplets from COVID-19 patients, these films do not last long from disinfection procedures. In addition, they become plastic waste in the end. According to news articles [2], the pandemic causes surges in global plastic waste and this trend could be permanent. For decades, plastic waste has been a significant issue in our society; therefore, the efforts to reduce plastic waste are necessary.

Among the aforementioned face shield materials, another candidate is CA film. In digital projector technology, CA is used in the manufacture of overhead projector transparencies. Unlike the aforementioned commercial face shield films, CA is a natural polymer, which is biodegradable and environmentally friendly. Therefore, CA waste can be buried in soil or water to safely decompose overtime [3]. Indeed, this is the major advantage of using CA as disposable face shield.

Although the face shield based on CA is commercially available, there are important considerations about the surface property of CA compared with other polymers such as hydrophobicity, optical transparency, antibacterial/antiviral property, and processability. Recent reports suggest that the hydrophobic coating on common surfaces may reduce the transmission of COVID-19 [4]. Therefore, the motivation of this research is how to improve the hydrophobicity of CA film while retaining the original optical transparency.

\section{Experimental}

The CA film (Transparency sheet, HP) and Hexamethyldisilazane (HMDS, electronic grade, Electron Microscopy Sciences) was purchased from Amazon.com and used as received. The CA film was treated with an oxygen plasma cleaner for 10 minutes to activate hydroxyl $\left(\mathrm{OH}^{-}\right)$groups in the CA surface. To coat the HMDS, the solution was drop-casted onto the CA surface and dried in the air for 30 minutes. The water contact angle was measured by a custom-built goniometer and the data was analyzed by using the software ImageJ [5].

\section{Results and Discussion}

The hydrophobicity of polymer films can be analyzed by the measurement of the contact angle with the water droplets on the surface. Based on literature reviews [6] [7] [8], Table 1 shows the static contact angle of three polymers mainly used in the manufacturing of face shields in the market. 
Table 1. Water contact angle (static) of common polymer films for face shield.

\begin{tabular}{cc}
\hline Polymer (Pristine) & Water contact angle (static) \\
\hline Polyethylene Terephthalate (PET) & $70.6^{\circ}-75.0^{\circ}[6][7][8]$ \\
Polycarbonate (PC) & $84.3^{\circ}-84.4^{\circ}[7][8]$ \\
Polyvinylchloride (PVC) & $72.0^{\circ}-76.3^{\circ}[6][7]$ \\
Cellulose Acetate (CA) & $\sim 55.0^{\circ}$ \\
\hline
\end{tabular}

In Table 1, the first three polymer films show slight hydrophilic character because their water contact angle is less than $90^{\circ}$ [9]. However, the CA film shows a higher degree of hydrophilicity; therefore, the hydrophobicity of CA films needs improvement. The typical image of a water droplet on the CA film used in this experiment is shown in Figure 1(a) (Pristine CA). In principle, the contact angle is estimated from the image between the horizontal surface and the tangent line from the left corner. The average contact angle from 10 different spots is $55.2^{\circ}( \pm 0.7)$. To promote the hydrophobicity of the CA film, a coating of HMDS, which is typically used in silicon microfabrication process to prepare hydrophobic surfaces, was applied. HMDS forms a hydrophobic layer from its abundance of methyl groups. In Figure 1(b), HMDS was drop-casted onto the pristine CA film without any treatment. After completely drying the HMDS solution in the air for $30-60$ minutes, the water contact angle was measured. However, unlike the expectation to improve the hydrophobicity of CA, the results indicated that the contact angle was similar to the pristine film, suggesting that HMDS coating is not effective.

In order to modify the pristine CA surface, an oxygen plasma treatment was utilized to oxidize the CA film. Previous reports [10] [11] suggested that oxygen plasma treatments can oxidize polymer surfaces and facilitate surface functionalization with various molecules. In fact, HMDS chemically binds its silicon atom to the oxygen by releasing ammonia moieties and then the surface can be stably modified by methyl groups [12]. After treating the CA film with oxygen plasma for 10 minutes, the improved wetting property was observed as shown in Figure 1 (c). The water contact angle was decreased to $43.7^{\circ}( \pm 3.3)$, suggesting that the CA surface was oxidized and became hydrophilic. The increase in hydrophilicity is due to the abundance of polar hydroxyl groups. The HMDS was then drop-casted onto the oxidized CA film and dried in the air. The water contact angle significantly increased to $77.1^{\circ}( \pm 3.3)$.

In Figure 1(e), the stability of HMDS was tested. The HMDS coated CA surface was stored under ambient conditions for one week and remained at a similar level of hydrophobicity at $78.8^{\circ}( \pm 3.8)$, indicating that the outside environment did not influence the properties of HMDS. The results clearly show that prior oxidation by oxygen plasma and subsequent HMDS modification can therefore improve the hydrophobicity of CA film in order to achieve water contact angles similar to other polymer films of face shields.

Another important property for the face shield is its optical transparency. Figure 2 shows the photograph of the CA films in three different surface treatments: 


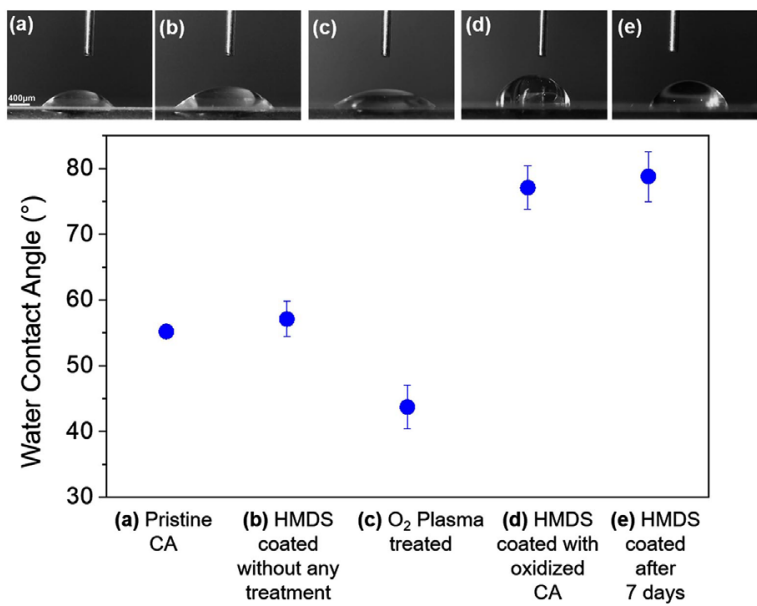

Figure 1. The water contact angle of each treatment: (a) pristine CA, (b) hydrophobic HMDS coating on pristine CA without prior oxidation, (c) after oxygen plasma treatment for 10 minutes, (d) HMDS coated on oxygen plasma treated CA film, and (e) HMDS coated CA after 7 days under ambient condition. Water contact angle was analyzed by custom-built goniometer.

\begin{tabular}{|c|c|c|}
\hline (a) Pristine CA & (b) Oxygen plasma treated CA & (c) HMDS coated CA \\
\hline & $\mathrm{Aa} \mathrm{Bb} \mathrm{Cc} \mathrm{Dd}$ & $\mathrm{Aa} \mathrm{Bb} \mathrm{Cc} \mathrm{Dd}$ \\
$\mathrm{Aa} \mathrm{Bb} \mathrm{Cc} \mathrm{Dd}$ & 1234567890 & 1234567890 \\
1234567890 & $\mathrm{Aa} \mathrm{Bb} \mathrm{Cc} \mathrm{Dd}$ & $\mathrm{Aa} \mathrm{Bb} \mathrm{Cc} \mathrm{Dd}$ \\
$\mathrm{Aa} \mathrm{Bb} \mathrm{Cc} \mathrm{Dd}$ & 1234567890 & 1234567890 \\
\hline 1234567890 & & \\
\hline
\end{tabular}

Figure 2. Transparency comparisons between: (a) pristine CA film, (b) $\mathrm{O}_{2}$ plasma treated CA film, and (c) HMDS coated CA film after $\mathrm{O}_{2}$ plasma treatment.

(a) the pristine CA film, (b) after 10 minutes of oxygen plasma, and (c) drop casted HMDS. There is no significant change in the optical transparency during the treatments; therefore, the CA modification process preserved the optical property of the film. For future research, spectrophotometer analysis should be performed to calculate the optical transparencies of each treatment.

\section{Conclusion}

The face shield has become a crucial element of PPE during the COVID-19 pandemic. The major material for the face shield is polymer films. There has been an increased attention to plastic waste because it is not environmentally friendly. Unlike common face shield polymer films, CA is a biodegradable natural polymer and one of the candidates for face shield manufacturing. However, the hydrophobicity of CA surface needs to be improved. The HMDS coating with oxygen plasma improved the CA film's hydrophobicity while retaining its optical transparency. Further research could include coating super-hydrophobic molecules, controlling porosity, and investigating antibacterial/antiviral activity.

\section{Acknowledgements}

S.L. thanks Professor Wonmo Kang in Arizona State University (Tempe, AZ, 
USA) for the research guidance and advice.

\section{Conflicts of Interest}

The author declares no conflicts of interest regarding the publication of this paper.

\section{References}

[1] Total Case Numbers and Death in the U.S. http://news.google.com/

[2] News Articles from $\mathrm{CNN}$ and $\mathrm{ABC}$ News. www.cnn.com/2020/05/04/world/coronavirus-plastic-waste-pollution-intl/index.ht ml,abcnews.go.com/Politics/covid-19-pandemic-concern-plastic-pollution-environ mental-headlines/story?id $=71698898$

[3] Ach, A. (1993) Biodegradable Plastics Based on Cellulose-Acetate. Journal of Macromolecular Science-Pure and Applied Chemistry, A30, 733-740. https://doi.org/10.1080/10601329308021259

[4] Siddiquie, R.Y., Agrawal, A. and Joshi, S.S. (2020) Surface Alterations to Impart Antiviral Properties to Combat COVID-19 Transmission. Transactions of the Indian National Academy of Engineering, 5, 343-347. https://doi.org/10.1007/s41403-020-00096-9

[5] Schneider, C.A., Rasband, W.S. and Eliceiri, K.W. (1997-2018). https://imagej.nih.gov/ij/

[6] Wu, W., Wu, J., Kim, J.H. and Lee, N.Y. (2015) Instantaneous Room Temperature Bonding of a Wide Range of Non-Silicon Substrates with Poly(Dimethylsiloxane) (PDMS) Elastomer Mediated by a Mercaptosilane. Lab on a Chip, 15, 2819-2825. https://doi.org/10.1039/C5LC00285K

[7] Wu, J. and Lee, N.Y. (2014) One-Step Surface Modification for Irreversible Bonding of Various Plastics with a Poly(Dimethylsiloxane) Elastomer at Room Temperature. Lab on a Chip, 14, 1564-1571. https://doi.org/10.1039/c3lc51324f

[8] Tang, L. and Lee, N.Y. (2010) A Facile Route for Irreversible Bonding of Plastic-PDMS Hybrid Microdevices at Room Temperature. Lab on a Chip, 10, 1274-1280. https://doi.org/10.1039/b924753j

[9] Law, K.Y. (2014) Definitions for Hydrophilicity, Hydrophobicity, and Superhydrophobicity: Getting the Basics Right. Journal of Physical Chemistry Letters, 5, 686-688. https://doi.org/10.1021/jz402762h

[10] Hetemi, D. and Pinson, J. (2017) Surface Functionalisation of Polymers. Chemical Society Reviews, 46, 5701-5713. https://doi.org/10.1039/C7CS00150A

[11] Dobos, A.M., Stoica, I., Olaru, N., Olaru, L., Ioanid, E.G. and Ioan, S. (2012) Surface Properties and Biocompatibility of Cellulose Acetates. Journal of Applied Polymer Science, 125, 2521-2528. https://doi.org/10.1002/app.36361

[12] Commercial Website.

http://microchemicals.com/products/adhesion promotion/hmds.html 